Meta

Journal des traducteurs

Translators' Journal

\title{
Pour les jargons
}

\section{Loïc Depecker}

Volume 39, numéro 4, décembre 1994

Hommage à Bernard Quemada : termes et textes

URI : https://id.erudit.org/iderudit/002539ar

DOI : https://doi.org/10.7202/002539ar

Aller au sommaire du numéro

Éditeur(s)

Les Presses de l'Université de Montréal

ISSN

0026-0452 (imprimé)

1492-1421 (numérique)

Découvrir la revue

Citer cet article

Depecker, L. (1994). Pour les jargons. Meta, 39(4), 736-740.

https://doi.org/10.7202/002539ar

\section{Résumé de l'article}

Bernard Quemada ouvre dans son œuvre des voies de recherche sur les questions de. terminologies techniques ou scientifiques, sur les vocabulaires d'ateliers ou de chantiers, sur les vocabulaires de métiers en général. Sa réflexion permet, notamment, de mettre en lumière la notion de jargon et de contribuer à la revaloriser. En effet, les jargons ayant été relégués à l'état de bas langage, ils n'ont pas encore, à ce jour, été suffisamment étudiés. Cet aspect de sa pensée peut avoir des incidences pratiques sur l'aménagement des vocabulaires de spécialités.
Tous droits réservés @ Les Presses de l'Université de Montréal, 1994
Ce document est protégé par la loi sur le droit d'auteur. L'utilisation des services d'Érudit (y compris la reproduction) est assujettie à sa politique d'utilisation que vous pouvez consulter en ligne.

https://apropos.erudit.org/fr/usagers/politique-dutilisation/ 


\title{
POUR LES JARGONS
}

LOÏC DEPECKER

Délégation générale à la langue française, Paris, France

\begin{abstract}
Résumé
Bernard Quemada ouvre dans son cuvre des voies de recherche sur les questions de. terminologies techniques ou scientifiques, sur les vocabulaires d'ateliers ou de chantiers, sur les vocabulaires de métiers en général. Sa réflexion permet, notamment, de mettre en lumière la notion de jargon et de contribuer à la revaloriser. En effet, les jargons ayant été relégués à l'état de bas langage, ils n'ont pas encore, à ce jour, été suffisamment étudiés. Cet aspect de sa pensée peut avoir des incidences pratiques sur l'aménagement des vocabulaires de spécialités.
\end{abstract}

\begin{abstract}
Bernard Quemada's seminal studies have opened the way for research into questions of technical and scientific terminologies, the vocabularies of the workshop or the building site, and trade and professional vocabularies in general. His works lead us to reappraise the idea of jargon and to give it its true value. Jargons had been relegated to a lower level of language and had not received the scholarly attention they deserve. This aspect of his thought can well be put to practical use in language planning for specialized vocabularies.
\end{abstract}

Dans son article «Technique et langage», Bernard Quemada retrace les grandes lignes des vocabulaires spécialisés du français depuis près d'un millénaire, selon une vue large qui embrasse une multiplicité de métiers. Ce texte est en bien des points novateur, et ouvre de larges voies: continuité de la langue technique avec la langue de tous les jours, distinction entre terminologies techniques et terminologies scientifiques, importance des parlers d'atelier ou de chantier, rôle de l'image dans la formation des vocabulaires de spécialité, description des évolutions, des modes de formation, méthodes de reconstruction, matériaux de référence, etc. L'article offre une profusion d'idées, dont celle de la problématique des jargons que nous retiendrons ici dans une perspective sociologique orientée vers l'aménagement des vocabulaires de spécialité.

Bernard Quemada écrit en une remarquable synthèse :

«Les vocabulaires des techniques français se sont constitués en même temps que ceux de la langue générale, et par des voies similaires, alors que les terminologies scientifiques en langue française apparaissent très timidement aux XIV et XVe siècles, progressent aux $X V I^{e}$ et $\mathrm{XVII}{ }^{\mathrm{e}}$, mais ne se dégagent tout à fait du latin qu'à partir du milieu du XVIII' ${ }^{\mathrm{e}}$. La confusion qui règne aujourd'hui entre les deux, en dépit de différences rigoureuses observables dès les origines, est d'autant plus difficile à corriger qu'elle s'est trouvée fondée sur la caution des linguistes puristes permettant pratiquement d'escamoter les usages professionnels du chantier ou de l'atelier. La simplification acquise, il devenait possible de traiter comme un ensemble cohérent le vocabulaire scientifique et la partie "noble" de celui des techniques (c'est-à-dire la plus générale). Si nous sommes attentif à éviter que le lecteur ne tombe dans le piège d'une organisation hiérarchisée des données, c'est que celui-ci a déjà fonctionné pour imposer la conviction que, du XVII ${ }^{\mathrm{e}}$ au XIX ${ }^{\mathrm{e}}$ siècle, maîtres et compagnons, puis contre-maitres et ouvriers, ne disposaient pas d'une langue mais d'une forme bâtarde, du type argot ou jargon. Il est exact que le caractère direct et naturel de leurs usages les apparentait au français populaire dont ils s'étaient principalement nourris, mais nous souffrons encore du point de vue sélectif qui, à ce titre, interdisait même d'en rendre compte. On comprend dès lors pourquoi, 
placé au rang des "bas langages", le parler des artisans et des "méchaniques" voisina longtemps avec le poissard des harangères et l'argot des malfaiteurs. On affecta de ne pas le comprendre ou de l'ignorer, comme en témoigne Furetière: "un mathématicien [= un ingénieur] pour parler de l'appuy d'un levier a recours au grec, et l'appelle hypomoclion, parce qu'il ne sait pas le nom François que luy donnent les ouvriers qui l'appellent orgüeil, et ainsi des autres" (Factums). Et quoi qu'en disent certains "défenseurs" de la langue, c'est bien cette image d'un "bon usage" aristocratique qui s'est trouvée transmise jusqu'à nos jours. Au seuil du $\mathrm{XX}^{\mathrm{e}}$ siècle, de grandes encyclopédies diffusaient encore les mêmes jugements de valeur: "Si les nomenclatures du chimiste et du médecin peuvent revendiquer le nom de langues et soutenir leur prétention avec quelque apparence de raison, le langage technique du plumassier ou celui du tailleur d'habits sont de véritables argots" (Brunetière).»

La perspective est ici largement tracée, et si l'on veut revenir sur la déclaration de Brunetière, nul doute qu'il faille resituer le débat. On peut concevoir en effet qu'à l'époque de Brunetière les parlers de l'atelier ou du chantier purent être parfois à ce point incompréhensibles pour un observateur extérieur qu'ils passaient pour des modes d'expression à part, ravalés, en l'absence de reconnaissance sociale forte de leurs usagers, à l'état de bas langage. Plusieurs métiers ont sans nul doute longtemps gardé une forte tradition d'argot, quand ce n'est celle de parlers cryptiques, comme peut l'être encore aujourd'hui celui des bouchers. Et si l'on entend par jargon le parler éventuellement argotisé d'un corps de métier, force est de constater qu'il serait bien rare aujourd'hui de rencontrer un tel parler au point qu'il représentât une langue à part, incompréhensible pour un auditeur extérieur. Sauf à relever bien sûr, dans une langue française quasiment usuelle, divers mots ou expressions pouvant éventuellement relever d'un usage spécialisé, familier ou argotique. Le temps semble passé où l'on pouvait encore entendre des chauffeurs de taxi parler en argot parisien, et l'écoute des routiers par exemple ne révèle que certains mots ou expressions à valeur cryptique ou ironique, tels les papas 22 pour les policiers, les $Y L$ (sans doute à l'origine young lady) pour les femmes, la tirelire pour le péage, la belle mère pour la seconde remorque, les boudins pour les pneus, etc. Rien de plus que des termes imagés, relevant parfois de l'argot général (comme le grand ruban pour l'autoroute). Et si le tailleur dit par exemple qu'il accessoirise un vêtement (il choisit chaussures, ceintures ou bijoux), ou s'il parle du flou (pour atelier de flou), désignant par là usuellement l'atelier de création, il ne s'agit là tout au plus que de mots techniques qui pour l'un actualise une possibilité morphologique de la langue, et pour l'autre n'est qu'un emploi légèrement métaphorisé du mot flou. Rien, en tout cas, d'un véritable argot.

Mais là où il y a persistance, c'est dans l'appréciation idéologique de ce genre de parler. Les termes de chantier et d'atelier continuent d'être considérés comme relevant d'un monde linguistique à part, nullement digne d'intérêt, comme la vie (l'avis) de ceux qui les utilisent. Et l'on pourra par exemple insister sur la traduction officielle de bulldozer en bouteur, sans jamais prendre en compte que ce qui se dit sur chantier est tout autre chose: un bul. L'homologation au Journal officiel du terme visu (pour visuel) dans le domaine informatique représente a contrario un cas unique de blanc-seing donné à un terme de jargon de métier (un jargonisme?). Cette stigmatisation ne touche pas seulement les métiers dédaignés, mais aussi par exemple le monde des laboratoires scientifiques: on ne trouvera pas dans un dictionnaire l'expression faire une manip (une manipulation), et a fortiori le verbe maniper, alors que ce sont pourtant là des termes qui s'emploient tous les jours. Le jugement de valeur dépréciatif qui touchait auparavant l'ensemble d'un parler de métier, a donc subsisté pour continuer de stigmatiser aujourd'hui les termes particuliers du chantier, de l'atelier, ou du laboratoire en référence souvent, il est vrai, à la pertinence morphologique sensée caractériser le bon français. Ce comportement induit évidemment bien des effets, dont le premier, double du mépris, est celui de l'ignorance. Alors que ces termes 
ou expressions de métier puisent souvent, profondément, dans l'histoire de la langue, les matériaux n'en sont même pas relevés, donc expliqués. Ainsi ne trouvera-t-on pas non plus facilement un terme pourtant relevé à plusieurs reprises lors de récentes enquêtes terminologiques de terrain, celui de haut-le-pied, alors même qu'il traverse plusieurs métiers. Sa formation peut paraître étrange, et seule une recherche comparative approfondie peut fournir la clé de son origine. On dit par exemple à la SNCF qu'une locomotive est hautle-pied lorsqu'elle circule sans wagons. Le terme se retrouve à la RATP pour désigner le fait qu'un véhicule ou un train ne s'arrêtent pas à un arrêt. L'une des étymologies populaires, promptement donnée par les techniciens, est que l'on ne freine pas à cet endroit, et que l'on garde ainsi le pied haut. Rien de convaincant cependant, et l'on voit la filiation sémantique entre l'usage de la SNCF et celui de la RATP. Le terme se trouve pourtant chez Littré, ce qui prête à penser qu'il puise bien avant dans l'histoire de la langue, comme paraît l'indiquer sa morphologie (sur le modèle de haut-le-corps, haut-le cul, etc.), et cela d'autant plus que plusieurs anciens termes de manège sont construits de cette façon, tel haut-la-main (tenir haut les rênes). Littré écrit de façon éclairante: «Haut le pied, en levant le pied [...] Par extension, haut le pied, sans être chargé. Renvoyer des chevaux haut le pied, les renvoyer sans être attelés ni montés. / Substantivement, un haut le pied, un cheval qui n'est pas attelé [...] Terme de chemin de fer et de messagerie. Train haut le pied, train vide de voyageurs.» Voilà qui lie les deux emplois relevés lors des enquêtes terminologiques: aller non chargé induit qu'il n'y a pas nécessité à marquer tel ou tel arrêt. Il y a donc eu, des transports à cheval et de l'hippomobile jusqu'à nous, continuité d'expression et d'emploi, à l'insu aujourd'hui des principaux intéressés, les spécialistes. Et c'est une constante qui se dégage dans le domaine des transports, que la filiation de la civilisation du cheval à celle des transports modernes (freiner, brider un moteur, etc.). Les techniciens sont passionnés par ces reconstructions, et sans doute les historiens de la langue leur doivent-ils explication sur leurs manières de parler, de penser, d'appréhender leur monde de travail. Car il y a des constantes dans les mentalités, qui offrent à l'historien, au sociologue, au psychologue, voire au psychanalyste, des voies de recherches encore inexplorées: dans plusieurs métiers par exemple, l'une des images principales de la sanction ou du passage en conseil de discipline est celle de la cuisson à feu plus ou moins vif, prolongement d'anciens supplices... Le tribunal des taxis est ainsi surnommé dans le métier la côtelette, lieu du passage au gril; de même, l'inspection générale de la police est la maison bouf carotte, où l'on fait mijoter, semble-t-il, à feu plus doux. L'image du jeu, donc de l'arbitraire, se retrouve également, par exemple à la RATP et à la SNCF : le conseil de discipline y est le tapis vert.

La psychanalyse aurait là également un beau terrain de recherche : les corporations les plus joyeuses ou les plus ironiques sont souvent celles dont le travail est le plus pénible: le rire pour surmonter l'angoisse. Ainsi les situations particulières suscitent ce que l'on pourrait appeler des «surmots»: descendre en égouts pour les égoutiers se dit certes descendre, aller dans le tube, mais aussi plonger: et c'est en fait deux fois plonger: sous terre et sous l'eau. Certains objets mobilisent également l'imaginaire, comme pour les gens de théatre, la douche : projecteur qui tombe verticalement sur la scène en un faisceau concentré qui ressemble à celui d'une douche, remarquable alliance, dans ce mot de métier, de l'eau et de la lumière. Également, certains personnages prêtent à surnoms, voire surmots: le patron, omniprésent dans la pensée des gens au travail, est le chef, le boss, le vieux, le pacha (dans la marine), ce dernier mot alliant avec une certaine ironie exotisme et vie tranquille au fil de l'eau. Sans parler d'images récurrentes, comme celle de la gamelle, surmot sans doute d'origine culinaire (l'instant de manger rompt providentiellement le rythme du labeur) pour désigner le casque de motard, du projecteur, du compteur électrique, etc. 
Il y a là tout un chantier encore inexploré qui mériterait d'être ouvert, ne serait-ce que pour l'histoire de la langue et pour la connaissance de la psychologie profonde des métiers. Mais aussi, et ce ne serait pas là seulement conquête linguistique, pour réhabiliter les jargons, mot qui devrait être au contraire considéré comme un mot noble: le jargon n'est en fait rien d'autre que l'inventivité des gens au travail. Et foin des belles tournures chantournées: la préface de l'Encyclopédie (1751), le dit à sa façon: «Dans un atelier, c'est le moment qui parle, non l'Artiste.» Sans doute ne serait-il pas vain de revenir sur certaines idées reçues, que véhicule l'idée même de jargon: langue basse, le jargon semble ne même pas avoir d'utilité propre. Alors qu'un ouvrier, un technicien, un scientifique n'utilisent pas un mot pour un autre. La précision de la terminologie double et complète la précision du geste technique.

Mais la réhabilitation des «jargons» peut aussi s'imposer d'autant plus aujourd'hui pour des raisons d'aménagement terminologique. Pris dans la pensée que des organismes de politique linguistique, État ou académies, ou que de toute façon quelqu'un est là pour dire comment il faut parler, donc se conduire, les gens au travail ne semblent pas, en France, croire qu'en fait c'est à eux-mêmes d'inventer la langue, et en priorité la leur propre. D'où sans doute une perméabilité encore plus grande à l'anglais qui, s'il s'introduit certes avec l'apport de nouvelles techniques, informatique et automatisation, trouve de plus des esprits perméables à son intrusion. Certes, l'anglais est souvent incrusté dans l'objet technique, ce qui induit des comportement linguistiques anglicisés, comme le montrent les termes de jargons anglicisés (les jarglicismes?) qui forment parfois de curieux assemblages: se loguer (to log) dit-on en informatique pour «se brancher»; opérer un satellite (to operate) signifie en spatiologie «l'exploiter»; la réconciliation de bagage (reconciliation) est dans le domaine aérien le fait, pour un passager, d'aller reconnaître son bagage avant le départ d'un avion; ou plus morphologiquement redoutable se faire five o'clocké qui exprime pour un trader le fait de ne pouvoir se débarrasser en temps voulu de la cargaison sur laquelle il a spéculé. Ainsi, un environnement anglicisé mène à une anglicisation de la langue, mais aussi la perméabilité des métiers à ce phénomène peut s'expliquer par la sorte d'inhibition psychologique qui consiste en France, souvent, à ne pas oser créer les mots. Réhabiliter les jargons pourrait conduire à changer les attitudes d'esprit, ce qui, dans l'aménagement raisonné d'une langue, est sans doute ce à quoi viser en priorité. Que chacun se sente responsable de sa langue, au même titre que de sa condition de travail, et le monde pourrait changer.

Enfin, pour l'aménageur de langue et le terminologue, il y a à l'évidence beaucoup à puiser dans les mots des métiers pour comprendre la manière dont les mots et les idées circulent, quel en est l'usage réel, et comment les ouvriers, techniciens, ou scientifiques peuvent réagir à des mesures d'ordre linguistique. Une visite en laboratoire suffit à montrer que les scientifiques, s'ils considèrent avoir besoin de terminologies harmonisées, ne souhaitent nullement se les voir imposer. Nul doute aussi que les parlers des métiers constituent une sorte de trésor où aller puiser le cas échéant, car bien des situations n'ont pas attendu les vagues d'anglicisation pour être décrites. Ainsi n'allait-on pas, dans l'armée, $\grave{a}$ un briefing, mais au rapport, et l'on dit encore couramment à la RATP que l'on va $\grave{a}$ l'instruction. Sans qu'il y ait là à porter nécessairement de jugement de valeur, n'y a-t-il pas lieu de prendre en considération ce genre de constance, ne serait-ce que pour ne pas être par trop enclin à penser qu'un nouveau monde commence avec l'anglais ?

Au-delà, la connaissance des parlers de métier peut contribuer à mieux illustrer une idée force de notre temps, qui est celle de la culture d'entreprise; qui n'est après tout que la conscience d'appartenir à une communauté de travail, avec ses traditions, ses modes de pensée, ses savoir-faire, sa grandeur. Et cette communauté se reconnaît aussi à travers ses mots, qui fondent une connivence. Sans doute, les entreprises et organismes qui cherchent 
aujourd'hui à mieux communiquer et à mettre en valeur la notion de culture d'entreprise, pourraient avoir avantage à mettre en valeur leur parler, et par là la conscience solidaire des gens au travail. L'idée suit son chemin, sur le fil de la sensibilisation faite par les enquêtes linguistiques ou terminologiques de terrain. Ainsi, cette publicité de la RATP, parue dans le Monde du 9 juin 1994, qui met en valeur les mots du métier, garants de la maîtrise technique: «Son nom: le "visiteur". Son but: assurer votre sécurité sous cette terre. Sa mission: traquer la moindre faille dans le réseau. Son arme: la perche à boule. Les visiteurs d'ouvrage de la RATP parcourent nuit et jour les $354 \mathrm{~km}$ du réseau métro et RER. Munis de leur perche à boule, ils sondent les couloirs et les tunnels et veillent au bon état des infrastructures pour vous assurer une sécurité constante et maximale. On n'en fera jamais assez pour vous donner l'esprit libre.»

\section{RÉFÉRENCE}

QUEMADA, Bernard (1978) : «Technique et langage», Histoire des techniques, Encyclopédie de la Pléiade, Paris, pp. 1146-1240. 